\title{
Molecular Simulations of Nanographene Systems
}

\author{
O. G. Ziogos ${ }^{\mathrm{a}, *}$, D. N. Theodorou ${ }^{\mathrm{a}}$ \\ ${ }^{a}$ School of Chemical Engineering, National Technical University of Athens, Zografou Campus, GR-15780 Athens, Greece
}

\begin{abstract}
Discotic polyaromatic hydrocarbons constitute a very promising materials family for organic electronic applications. Its members can be considered as finite graphene flakes of nanometric dimensions. In this work, a plethora of multiscale simulations are employed for the determination of electronic, structural and dynamical properties of nanographene molecules that bear around their periphery flexible functional groups and tend to form well-organized molecular wires and crystals. Charge transfer properties are examined at the molecular dimer level and relationships between internal molecular wire structure and material properties are elucidated.

(C) 2017 Elsevier Ltd. All rights reserved.

Selection and/or Peer-review under responsibility of 11th Panhellenic Scientific Conference on Chemical Engineering.

Keywords: graphene; nanographene; molecular wire; charge transfer; molecular mechanics; polyaromatic stacking; molecular dynamics
\end{abstract}

\section{Introduction}

Discotic molecules comprised by $s p 2$ hybridized carbon atoms constitute a very promising materials family for organic electronic applications [1]. Due to the interaction of neighboring delocalized pi molecular orbitals, semiconducting behavior via charge hopping mechanisms is manifested when such molecules are neatly packed. A highly interesting characteristic of these molecules relies on their ability to host flexible side groups about their periphery via covalent functionalization. This peripheral alteration may lead to appreciable solubility in common organic solvents, thus prompting the use of "wet chemistry" methods for synthesis and processing towards the fabrication of organic electronic devices with minimum cost compared to traditional inorganic electronics.

Prototype discotic molecules with semiconducting properties are those consisting of fused phenyl rings, such as triphenylene, perylene and coronene derivatives and more "exotic" molecules like metallomesogens from the

* Corresponding author. Tel.: +30-210-772-3216; fax: +30-210-772-3112.

E-mail address: oziogos@mail.ntua.gr

2214-7853 @ 2017 Elsevier Ltd. All rights reserved.

Selection and/or Peer-review under responsibility of 11th Panhellenic Scientific Conference on Chemical Engineering. 
porphyrin and phthalocyanine groups and macrocyclic molecules based on phenylacetylene, alongside with a phethora of other related aromatic molecules [2].

After the peripheral covalent functionalization utilizing flexible side groups, such molecules gain enhanced spatial mobility and self-organize into molecular wires that, in turn, form symmetric molecular crystals. Semiconducting behavior is manifested via charge hopping along the axial direction of the molecular wires.

The current flagship discotic molecule for organic electronic applications is hexa-peri-hexabenzocoronene (HBC), a molecule with hexagonal symmetry, made up of thirteen fused benzoic rings. Its dodecyl substituted variant (HBC-C12) exhibits in its crystalline form charge carrier mobility up to one third of graphite's perpendicular to the graphitic sheet [3]. A special feature of HBC-C12 is the manifestation of liquid crystalline behavior when heated above room temperature [4]. Liquid crystallinity is exhibited as an en-masse sliding of the molecular wires parallel to the axial direction, with the latter forming a hexagonal supramolecular lattice.

The fusion of even more benzoic rings leads to the formation of extended flat polyaromatic molecules with variable symmetry and size. These structures can be considered as nanometric graphene segments that are formally referred to as nanographenes. Some characteristic examples are superphenylene (C96), consisting of thirty four benzoic rings and bearing trigonal symmetry, and the $\mathrm{C}_{132} \mathrm{H}_{34}(\mathrm{C} 132)$ nanographene of tetragonal symmetry, made up by fifty rings. These molecules can also be crudely considered as the fusion product of three and four HBC molecules respectively [5]. Through functionalization with aliphatic side chains, such as dodecane (C12) and phytane $\left(\mathrm{C}_{20} \mathrm{H}_{42}\right.$ or $\left.\mathrm{C} 16,4\right)$, adequate solubility is achieved that leads to the formation of molecular wires and ultimately - crystals of hexagonal symmetry [5]. The molecules under study are depicted in Fig. 1.
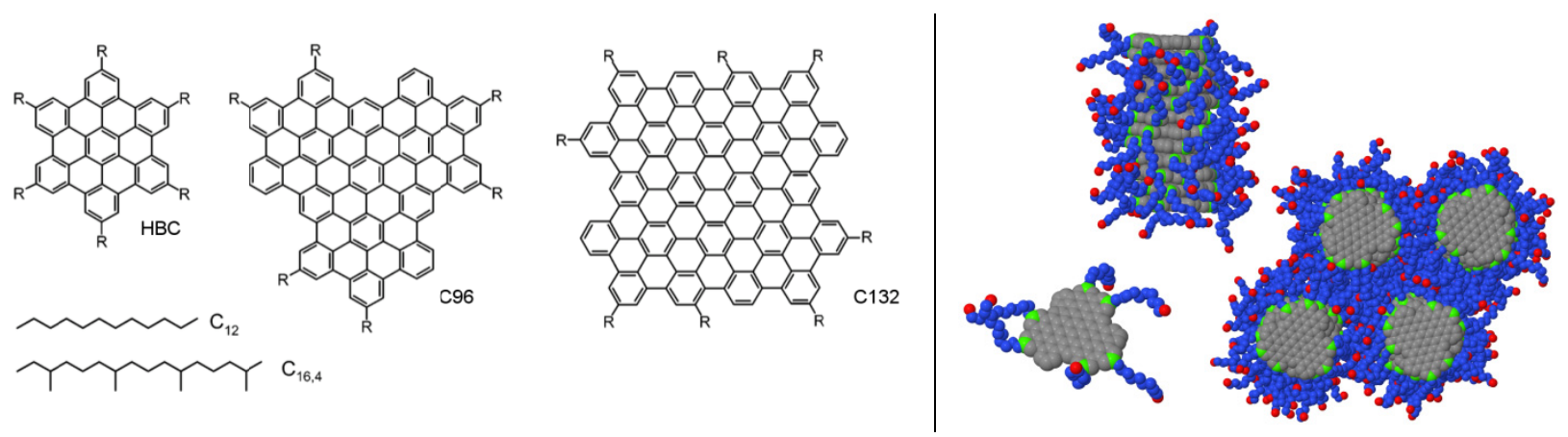

Fig. 1. Left panel. Nanographenes under study, alongside with appropriate side chains. Right panel. An illustration of the self-organization mechanism: from an isolated molecule to a molecular wire and - finally - to a molecular crystal.

The purpose of this work is the computational study of soluble C96 and C132 supramolecular assemblies. To be more precise, an analysis from the level of an isolated molecule to a molecular dimer, up to a molecular wire and a molecular crystal is carried out, utilizing both ab-initio methods and empirical atomistic simulations.

Special attention is focused on the preferable stacking patterns at the molecular dimer level, since the relative position of the molecules dictates the strength of the charge transfer mechanism [6]. Furthermore, the studied systems constitute a prototype paradigm of supramolecular nanostructures of cylindrical form with core-shell characteristics: the stacked polyaromatic cores define a periodic axial core surrounded by a soft aliphatic nanophase. Structural and dynamical investigations of such systems constitute an interesting case study in the field of computational nanotechnology.

\section{Computational Details}

The present computational study employs calculations at different length scales. Quantum mechanical ab-initio calculations using Density Functional Theory (DFT) are utilized in order to study isolated molecules and molecular dimers, aiming at the study of structural and electronic properties. All DFT calculations are carried out with the open-source package NWCHEM [7], using a Gaussian 6-31G** basis set for structural optimization and a 6-31G for charge transfer calculations, and the hybrid B3LYP [8,9] functional. At the molecular dimer level, the strength of the 
charge transfer near the Fermi level is quantified via the so-called Charge Transfer Integral (CTI), which is the Hamiltonian matrix element that couples the frontier molecular orbitals participating to the hopping process.

Furthermore, empirical Molecular Mechanics (MM) calculations are used at the dimer level, with each molecule bearing appropriate side chains, in order to deduce favorable stacking patterns. A series of molecular dimers is constructed through appropriate displacements and rotations. MM energy minimizations are carried out, resulting in the calculation of the dimerization energy. Having in mind the minimum energy stacking patterns from the aforementioned procedure, we construct initial configurations of periodic molecular wires and crystals that are equilibrated via Molecular Dynamics (MD) simulations.

In order to create plausible initial structures, unsubstituted polyaromatic cores are stacked in accordance to experimental data regarding the core and column distances [5]. Then, the aliphatic phase is grown via a bond-bybond fashion with Monte Carlo (MC) criteria [10] at room temperature. The conformational statistics of side chain backbone dihedral angles is of paramount importance for a realistic building procedure. To this end, based on published data on linear alkanes of low molecular weight [11], an $80 \%$ trans population is used. All MM and MD calculations are carried out using the LAMMPS package [12]. The integration of the equations of motion during MD simulations in the isothermal-isostress statistical ensemble is based on the Martyna-Tobias-Klein [13] algorithm, utilizing periodic boundary conditions. Mesh based techniques are employed in order to account for the periodic nature of long range interactions [14]. The quantification of particle interactions is accomplished through an empirical force field capable of capturing the properties of such materials [15].

\section{Results and Discussion}

As far as the structure of isolated molecules is concerned, the morphology of HBC, C96 and C132 molecules in their pristine and methyl substituted forms is studied via DFT calculations. For pristine cores, there is no deviation from polyaromatic planarity. The same conclusion applies for methyl substituted HBC and C96 molecules, since the linking sites are situated at such positions that do not trigger any steric hindrance. On the other hand, for methyl substituted $\mathrm{C} 132$, excluded volume intramolecular interactions at two counter diametrical linking sites cause local structural distortions that result in core bending, as depicted in Fig. 2. The overall bending is dependent on the relative position of the methyl groups with respect to the core plane, leading to two polymorphs of different curvature. The lowest energy polymorph corresponds to the configuration with both methyl groups on the same side with respect to the core, leading to a molecular structure with a more pronounced curvature. Nevertheless, the energy difference between the two polymorphs is $\sim 13 \mathrm{meV}$, allowing a crossover between them at room temperature.
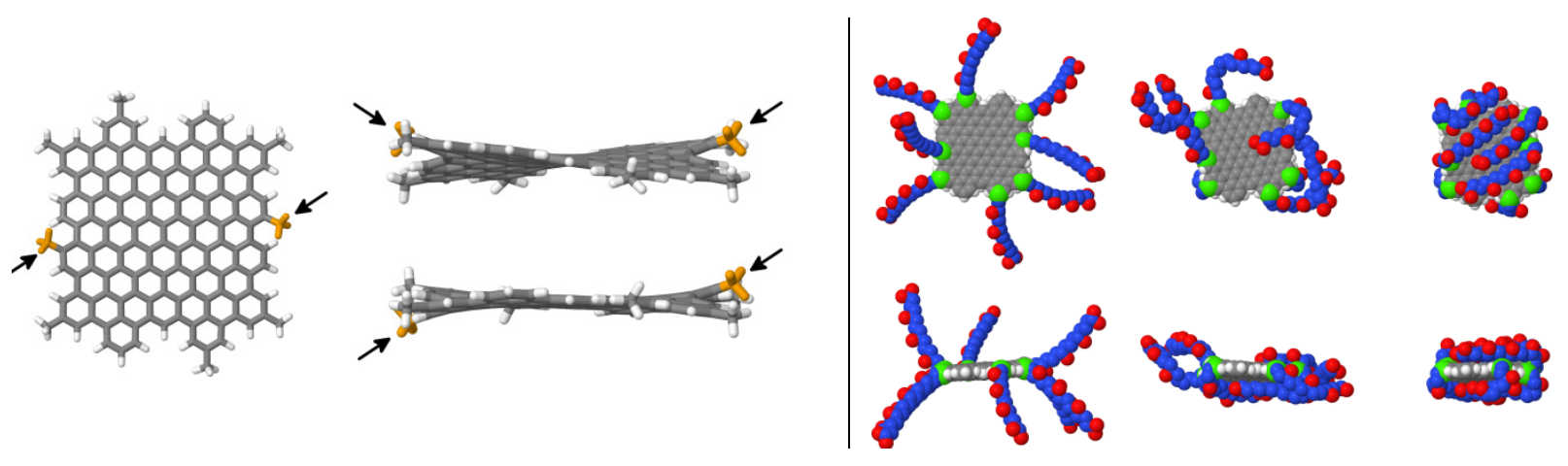

Fig. 2. Left panel. Top view of the methyl substituted C132 molecule and side views of the two polymorphs. The arrows highlight the counter diametrical methyl units that cause the curvature effect. Right panel. Depiction of C16,4 side chain folding onto a C132 core according to MD simulations at room temperature.

The folding mechanism for $\mathrm{C} 12$ and C16,4 side chains is probed via MD simulations for isolated cores. For every molecule, a sequence of gauche conformational defects near the linking site helps the side groups embrace the polyaromatic core in an ordered manner. This mechanism is illustrated in Fig. 2 for C132-C16,4.

Regarding the quantum charge transfer study of molecular dimers, the twist angle between parallel core molecules 
affects dramatically the value of the CTI. Calculations on coronene, HBC, C96 and C132 dimers suggest that the optimum charge transfer is accomplished for graphitic-like AA stacking patterns, with the twist angle being an integer multiple of 60 degrees. CTI values versus twist angle are shown in Fig. 3.

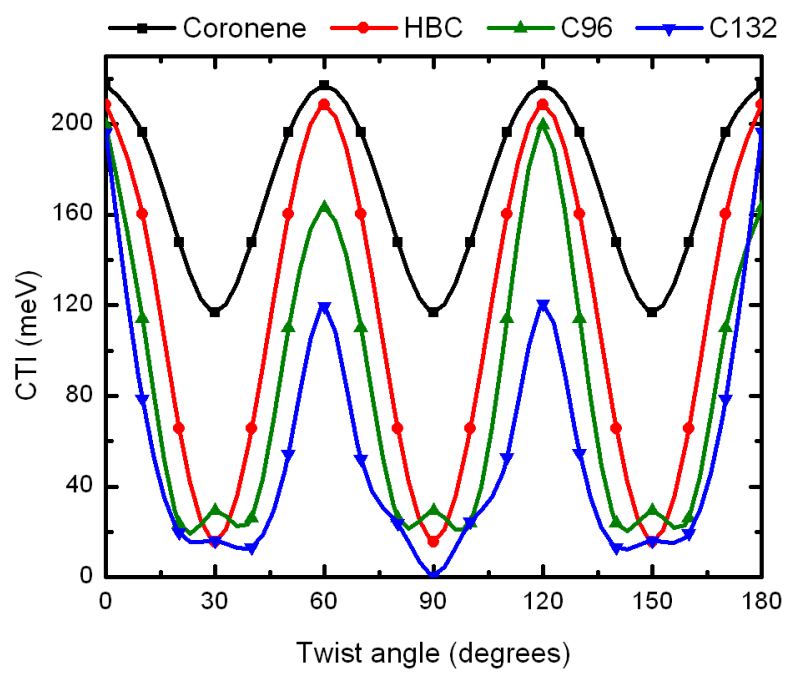

Fig. 3. Charge transfer integral values for hole transport versus twist angle.

Lowest energy stacking patterns are obtained via empirical MM calculations with all molecules bearing $n$-hexane side groups. HBC molecules exhibit two patterns: a parallel-displaced, graphitic-like motif, in accordance to experimental findings [16] and a "daisy-like" pattern of 30 degrees twist angle, also previously reported in the literature [17]. For C96 and C132, two and three favorable stacking patterns are identified. C96 shows a tendency to a 20 degrees twist angle, with the AA 60 degrees stacking lying at an elevated energy level. In the case of C132, the lowest energy landscape is more complicated, containing both twisted configurations (20 and 90 degrees) and a graphitic-like 60 degrees pattern.

Relying on the stacking information gained from MM dimer calculations, a series of molecular crystals is created. Crystals comprised of C96 core molecules are constructed with a 60 degrees twist angle and two variants based on the 20 degrees stacking motif: one helical and one alternating. In the case of $\mathrm{C} 132$, should symmetry be taken into account, five rotational profiles arise: three alternating with twist angle 20, 60 and 90 degrees and two helical for 20 and 60 degrees. Once the rotational state of the core molecules is established, a bond-by-bond MC growth scheme is utilized to create the aliphatic nanophase. All resulting systems are studied at room temperature and at an elevated temperature of $400 \mathrm{~K}$ via MD simulations.

Distributions of the twist angle between neighboring cores, alongside with time series of the tilt angle formed by a normal to the core vector and the axial direction are shown in Fig. 4 and Fig. 5. Systems with graphitic-like stacking patterns (60 degrees twist angle) tend to form wires with pronounced tilting. These structures are linked to the so-called "herringbone" patterns that are inherent to pristine [18] and substituted [4] HBC molecular crystals. Furthermore, C96 based systems tend to form 20 degrees twist angle configurations with rising temperature. The side chain thermal motion evolves quite slowly due to the dense nature of the aliphatic nanophase, effectively prohibiting a complete transition between stacking patterns via MD simulations. Nevertheless, the trend from the 60 degrees pattern to the 20 degrees pattern is evident. To consolidate this trend, isolated molecular wire MD simulations were carried out, exhibiting this transition within the time span available via MD simulations.

The dynamical response of the aliphatic nanophase is examined, aiming at a possible link between the stacking pattern and the side chain relaxation mechanism. To this end, side chain end-to-end vectors are calculated at equilibrium and their temporal evolution is quantified via the rotational autocorrelation function based on the second-order Legendre polynomial. All autocorrelation spectra relevant to side chain dynamics are depicted in Fig. 6. 

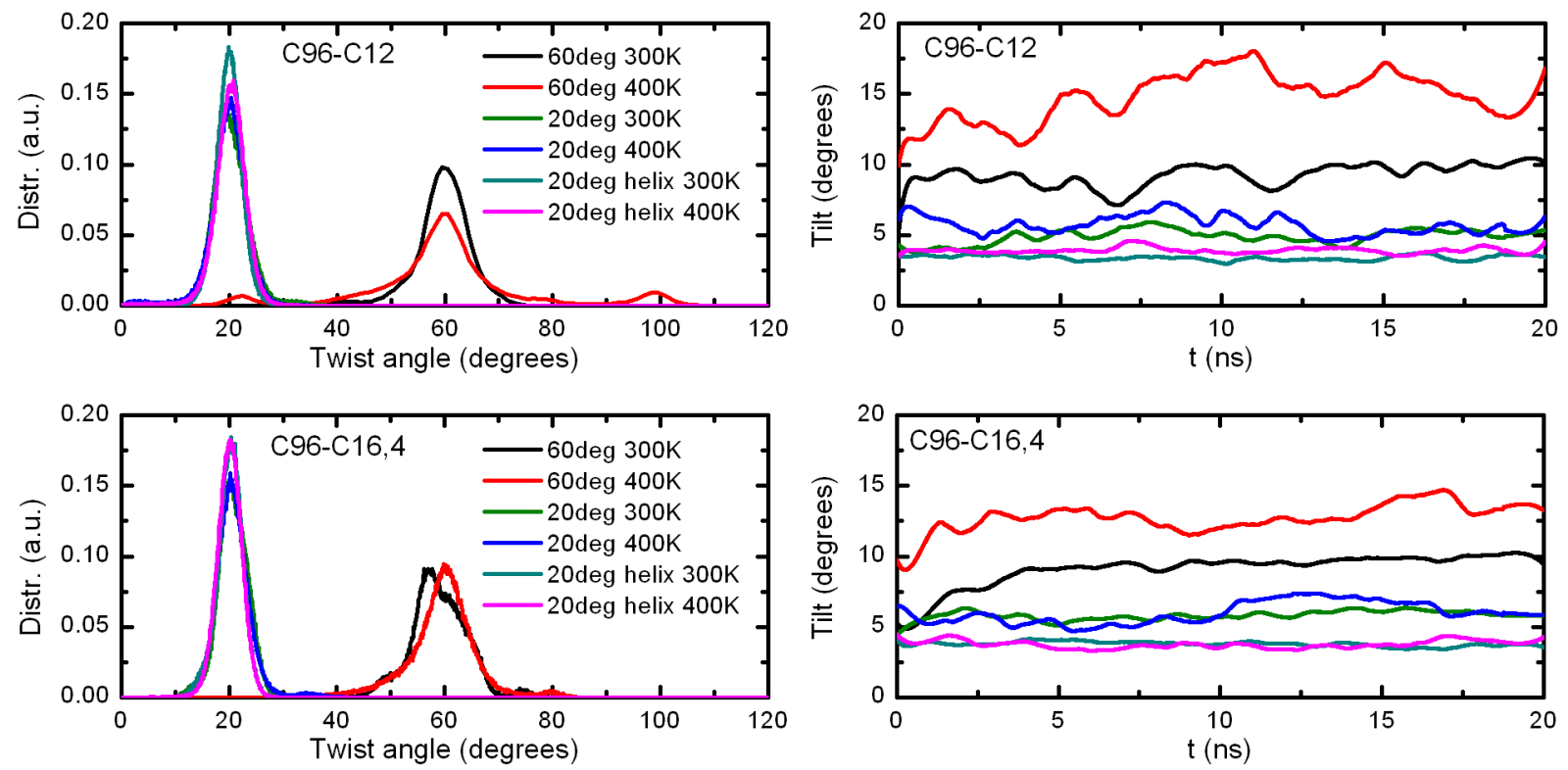

Fig. 4. Temperature dependence of twist angles (left) for C96-C12 and C96-C16,4 molecular crystals and time series of the tilt angle (right). The color coded legend of the left diagrams applies to the right ones as well.
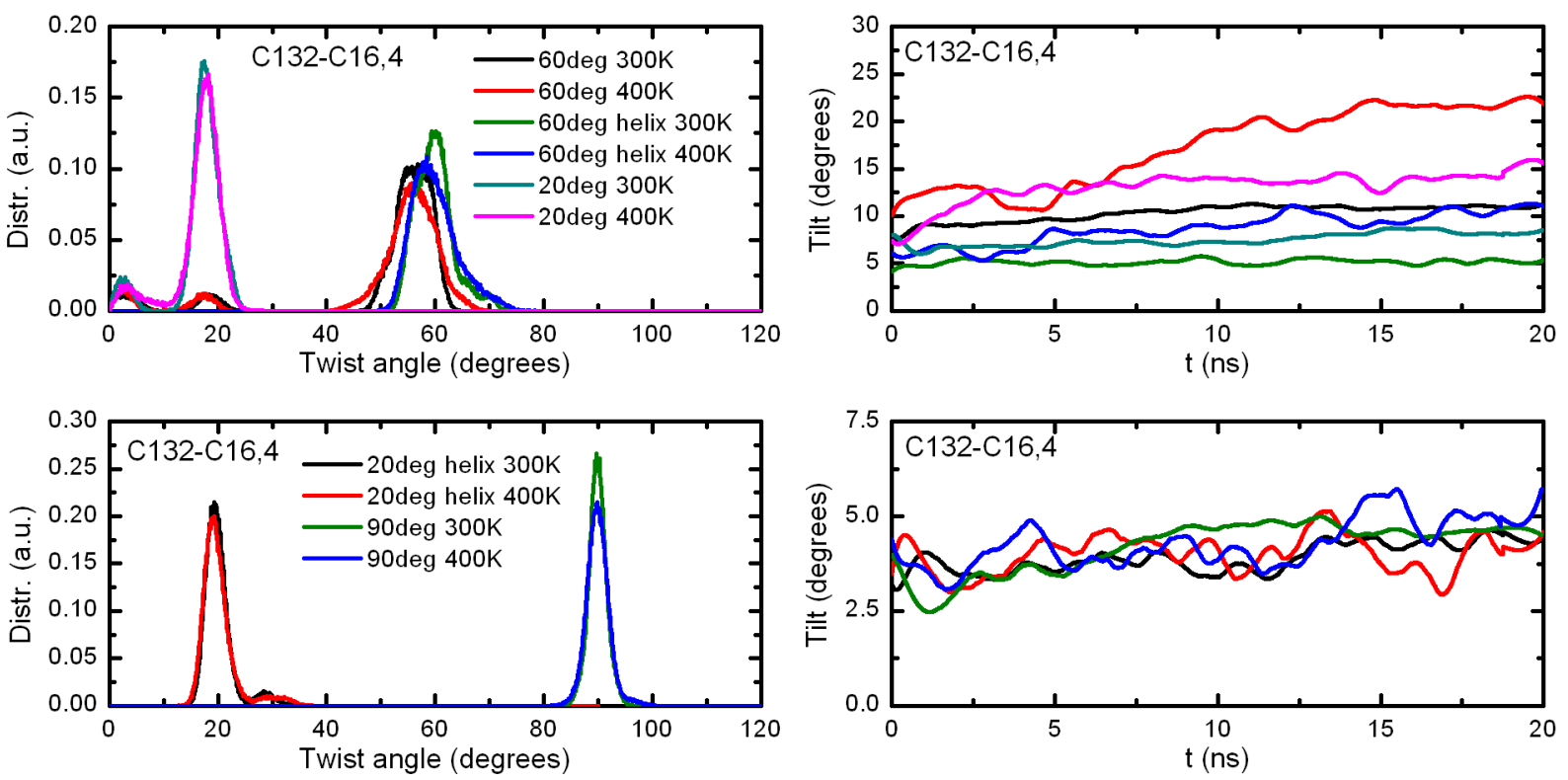

Fig. 5. Temperature dependence of twist angles (left) for C132-C16,4 molecular crystals and time series of the tilt angle (right). The color coded legend of the left diagrams applies to the right ones as well.

A well defined layering for the side chain relaxation mechanism is evident for C96 based molecular crystals at the temperature of $400 \mathrm{~K}$, with the helical polymorphs exhibiting faster decorrelation. This picture is more complicated in the case of C132-C16,4 crystals. Even so, a relative dependence of the relaxation mechanism on the internal wire structure is evident. 


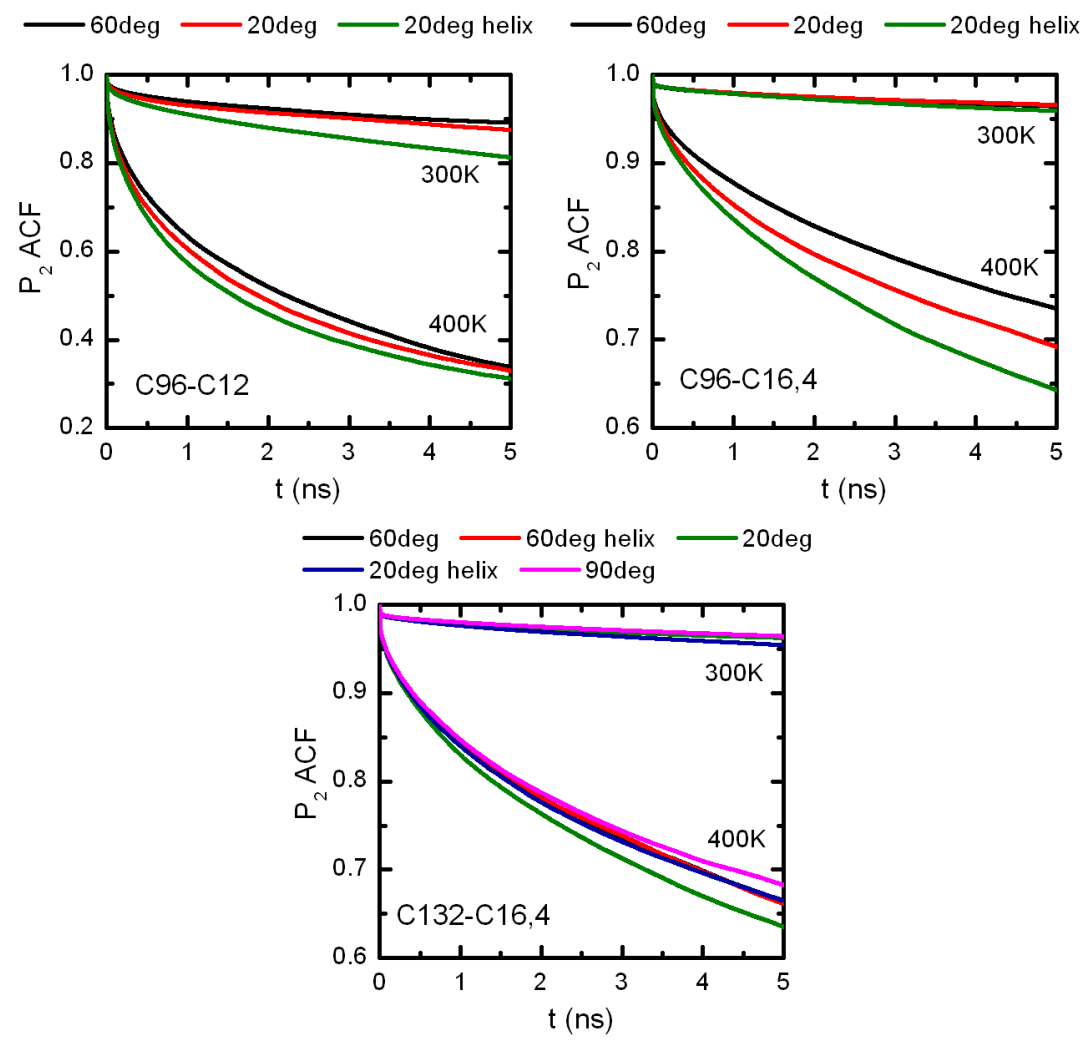

Fig. 6. Second-order Legendre polynomial time autocorrelation functions for the end-to-end side chain vectors for all studied systems.

\section{Conclusions}

Based on the findings of the present study, the internal structure of supramolecular wires comprised of substituted nanographene molecules seems to affect a series of material properties. As far as the charge transfer is concerned, there is a direct influence of the twist angle on the charge transfer integral, exhibiting maxima for graphitic-like AA stacking patterns. The morphology of the molecular cores gives rise to a series of possible stacking patterns, some of them hindering the charge transfer hopping mechanisms. Nevertheless, the structural robustness of substituted C96 and $\mathrm{C} 132$ systems, which is based on the fact that there is no thermotropic variation in structural properties, makes these materials a viable alternative to smaller core materials (e.g. HBC-C12) that exhibit conductivity variations due to temperature rise. Regarding the studied molecular crystals, there is a distinct trend as far as the tilt angle is concerned. Graphitic-like AA stacking patterns lead to tilted, herringbone-like configurations while the rest, "daisylike" patterns show small or even no tilting at all. Finally, studying such materials from a core-shell systems perspective, the relative positions from where the side chains protrude seem to affect the relaxation mechanism of the aliphatic nanophase surrounding the cores.

\section{Acknowledgements}

This research has been co-financed by the European Union (European Social Fund - ESF) and Greek national funds through the Operational Programme 'Education and Lifelong Learning' of the National Strategic Reference Framework (NSRF) - Research Funding Programme: THALIS. Investing in knowledge society through the European social fund [grant number MIS 379436] and by the IKY Fellowships of Excellence for Postgraduate Studies in Greece - Siemens Programme [award number 11126/13a]. This work was also supported by computational 
resources granted by the Greek Research \& Technology Network (GRNET) on the National HPC facility 'ARIS' under the projects 'Multiscale Simulations of Crystalline and Liquid-Crystalline Systems - MultiCLC', 'Hierarchical Modeling of Soft Matter - HMSM' and 'Molecular Simulations of Membrane-based Systems - MSMS'.

\section{References}

[1] J. Wu, W. Pisula, K. Müllen, Chem. Rev. 107 (2007) 718.

[2] S. Kumar, Chem. Soc. Rev. 35 (2006) 83-109.

[3] A.M. van der Craats, J.M. Warman, Adv. Mater. 13 (2001) 130-133.

[4] I. Fischbach, T. Pakula, P. Minkin, A. Fechtenkötter, K. Müllen, H.W. Spiess, J. Phys. Chem. B. 106 (2002) $6408-6418$.

[5] W. Pisula, Z. Tomović, C. Simpson, M. Kastler, T. Pakula, K. Müllen, Chem. Mater. 17 (2005) 4296-4303.

[6] X. Feng, V. Marcon, W. Pisula, M.R. Hansen, J. Kirkpatrick, F. Grozema, D. Andrienko, K. Kremer, K. Müllen, Nature Materials 8 (2009) 421-426.

[7] M. Valiev, E.J. Bylaska, N. Govind, K. Kowalski, T.P. Straatsma, H.J.J. van Dam, D. Wang, J. Nieplocha, E. Apra, T.L. Windus, W.A. de Jong, Comput. Phys. Commun. 181 (2010) 1477-1489.

[8] A.D. Becke, J. Chem. Phys. 98 (1993) 5648-5652.

[9] C. Lee, W. Yang, R.G. Parr, Phys. Rev. B 37 (1988) 785-789.

[10] D.N. Theodorou, U.W. Suter, Macromolecules 18 (1985) 1467-1478.

[11] L.L. Thomas, T.J. Christakis, W.L. Jorgensen, J. Phys. Chem. B 110 (2006) 21198-21204.

[12] S. Plimpton, J. Comp. Phys. 117 (1995) 1-19.

[13] G.J. Martyna, D.J. Tobias, M.L. Klein, J. Chem. Phys. 101 (1994) 4177-4189.

[14] R.W. Hockney, J.W. Eastwood, Computer Simulation Using Particles, Adam Hilger, New York, 1989.

[15] O.G. Ziogos, D.N. Theodorou, Mol. Phys. 113 (2015) 2776-2790.

[16] S.P. Brown, I. Schnell, J.D. Brand, K. Müllen, H.W. Spiess, J. Am. Chem. Soc. 121 (1999) 6712-6718.

[17] D. Mackie, G.A. DiLabio, J. Phys. Chem. A 112 (2008) 10968-10976.

[18] R. Goddard, M.W. Haenel, W.C. Herndon, C. Krüger, M. Zander, J. Am. Chem. Soc. 117 (1995) 30-41. 